\title{
AN EXTENSION OF THE ERDÖS-RÉNYI NEW LAW OF LARGE NUMBERS
}

\author{
STEPHEN A. BOOK
}

ABSTRACT. If $S_{n}$ is the $n$th partial sum of a sequence of independent, identically distributed random variables $X_{1}, X_{2}, \cdots$ such that $E\left(X_{1}\right)=0$ and $E\left(\exp \left(t X_{1}\right)\right)<\infty$ for some nonempty interval of $t$ 's, then, for a wide range of positive numbers $\lambda$, Erdös and Rényi (1970) showed that $\Sigma(N,[C(\lambda) \log N])$ converges with probability one to $\lambda$ as $N \rightarrow \infty$, where $\Sigma(N, K)$ is the maximum of the $N-K+1$ averages of the form $K^{-1}\left(S_{n+K^{-}} S_{n}\right)$ for $0 \leq n \leq N-K$, and $C(\lambda)$ is a known constant depending on $\lambda$ and the distribution of $X_{1}$. The objective of the present article is to state and prove the Erdös-Rényi theorem for the $N-K+1$ "averages" of the form $K^{-1 / r}\left(S_{n+K}-S_{n}\right)$, where $1<r<2$. This form of the Erdös-Rényi theorem arises from the extended form of the strong law of large numbers which asserts that, if $E\left(\left|X_{1}\right|^{r}\right)<\infty$ for some $r, 1 \leq r<2$, and $E\left(X_{1}\right)=0$, then $n^{-1 / r} S_{n}$ converges with probability one to 0 as $n \rightarrow \infty$.

0 . Introduction. If $\left\{X_{n}: 1 \leq n<\infty\right\}$ is a sequence of independent, identically distributed (i.i.d.) random variables, with partial sums $S_{n}=\sum_{k=1}^{n} X_{k}$, then there are $N-K+1$ successive averages of the form $K^{-1}\left(S_{n+K}-S_{n}\right)$, one for each value of $n$ between 0 and $N-K$, inclusive. We denote the largest of these averages by $\Sigma(N, K)$, i.e.

$$
\Sigma(N, K)=\max \left\{K^{-1}\left(S_{n+K}-S_{n}\right): 0 \leq n \leq N-K\right\}
$$

Then, by the Borel-Cantelli lemma, $P\left\{\lim _{N \rightarrow \infty} \Sigma(N, 1)=\mu\right\}=1$ if $\mu \leq \infty$ is the essential supremum of $X_{1}$. If $E\left(X_{1}\right)=0$, the strong law of large numbers asserts that $P\left\{\lim _{N \rightarrow \infty} \Sigma(N, N)=0\right\}=1$. What happens to $\Sigma(N, K)$ for values of $K$ between 1 and $N$ was the subject of the 1970 article [4] by Erdös and Rényi. If $X_{1}$ has moment-generating function $\phi(t)=E\left(\exp \left(t X_{1}\right)\right)<\infty$ for a sufficiently large interval of $t$ 's, then for every $\lambda$ between 0 and $\mu$ (exclusive

Presented to the Society, January 15, 1974; received by the editors November 5, 1973 and, in revised form, January 31, 1974.

AMS (MOS) subject clas sifications (1970). Primary 60F 15; Secondary 60F10.

Key words and phrases. Strong limit theorems, laws of large numbers, large deviations, moment-generating functions. 
of the endpoints), Erdös and Rényi showed that there exists a constant $C(\lambda)$, whose value can be determined, depending on $\lambda$ and the distribution of $X_{1}$, such that

$$
P\left\{\lim _{N \rightarrow \infty} \Sigma(N,[C(\lambda) \log N])=\lambda\right\}=1 .
$$

Here $[y]$ denotes the greatest integer $\leq y$. Erdös and Rényi called their result "a new law of large numbers" because of the fundamental relation it bears to the strong law of large numbers. The theorem in [2] extends the Erdös-Rényi result to the case of weighted sums of i.i.d. random variables.

It is the objective of the present article to extend the Erdös-Rényi theorem to averages of the form $K^{-1 / r}\left(S_{n+K}-S_{n}\right)$ for $1<r<2$. Toward this end, we define

$$
\Sigma_{r}(N, K)=\max \left\{K^{-1 / r}\left(S_{n+K}-S_{n}\right): 0 \leq n \leq N-K\right\} .
$$

Again $P\left\{\lim _{N \rightarrow \infty} \Sigma_{r}(N, 1)=\mu\right\}=1$ and, if $E\left(X_{1}\right)=0$ and $E\left(\left|X_{1}\right|^{r}\right)<\infty$, the strong law of large numbers asserts that $P\left\{\lim _{N \rightarrow \infty} \Sigma_{r}(N, N)=0\right\}=1$. The strong law of large numbers in the form $P\left(n^{-1 / r} S_{n} \rightarrow 0\right)=1$ for $1<r<2$ can be found in Loève's text [7, p. 243]. In the present article, we intend to show that, for every pos itive $\lambda, 0<\lambda<\infty$.

$$
P\left\{\lim _{N \rightarrow \infty} \Sigma_{r}\left(N,\left[\left(2 \lambda^{-2} \log N\right)^{r /(2-r)}\right]\right)=\lambda\right\}=1
$$

as long as there exists some nondegenerate interval of $t$ 's, containing the origin in its interior, for which $\phi(t)=E\left(\exp \left(t X_{1}\right)\right)<\infty$. Note that, in the present situation, $\phi(t)$ is required to exist only in a nondegenerate interval, not necessarily a sufficiently large one, and that the constant $2 \lambda^{-2}$ depends only on $\lambda$ and is independent of the distribution of $X_{1}$.

Erdös and Rényi based their proof on the large deviation theorem of Cramér [3], in the form due to Bahadur and Ranga Rao [1], which asserts that

$$
P\left(S_{n} \geq \lambda n\right) \sim(2 \pi n)^{-1 / 2} \rho^{n} b_{n},
$$

where $\rho=\exp (-1 / C(\lambda)),\left\{b_{n}: 1 \leq n<\infty\right\}$ is a bounded sequence, and the symbol " " indicates that the ratio of the two sides tends to 1 as $n \rightarrow \infty$. To provide a point of departure for the theorem of the present article, we need a large deviation theorem also, one due to Petrov [8], which can be found in the most easily usable form in the monograph of Ibragimov and Linnik [6]. Petrov's theorem, which is a generalization of and has the same form as the original Cramér theorem, implies in our situation, for $1<r<2$, that

$$
P\left(S_{n} \geq \lambda n^{1 / r}\right) \sim\left(2 \pi n^{\alpha}\right)^{-1 / 2} \lambda^{-1} \exp \left\{-1 / 2 \lambda^{2} n^{\alpha}(1+o(1))\right\}
$$


as $n \rightarrow \infty$, where $\alpha=(2-r) / r$. Note that if $r=1$, then $\alpha=1$, and the above statement is somewhat reminiscent of the Bahadur-Ranga Rao result.

Petrov's large deviation theorem and the corollary of it that we use are recorded in $\$ 1$. $\$ 2$ contains the extension of the Erdös-Rényi theorem. In $\$ 3$, we briefly compare the main theorem of this article with the original Erdös-Rényi theorem, focusing on the relationship between $C(\lambda)$ and $2 \lambda^{-2}$.

1. Petrov's theorem and its corollary. We consider throughout the paper a sequence of nondegenerate i.i.d. random variables $\left\{X_{n}: 1 \leq n<\infty\right\}$ such that $E\left(X_{1}\right)=0, \operatorname{Var}\left(X_{1}\right)=1$, and $\phi(t)=E\left(\exp \left(t X_{1}\right)\right)<\infty$ for $|t|<B$, where $0<B \leq \infty$.

For the theorem of the present article, we need no condition on the moment-generating function (m.g.f.) $\phi$ beyond its existence in a nondegenerate interval containing the origin in its interior. Bahadur and Ranga Rao, and therefore Erdös and Rényi, had to require that the function $Q(t)=\phi^{\prime}(t) / \phi(t)$ take on the value $\lambda$ at some point $t=t_{\lambda}$. (An analogous condition is required in the case of weighted sums that is studied in [2].) That assumption is vital in the ir situation, however, because $C(\lambda)$ turns out to be the reciprocal of $\lambda t_{\lambda}-\log \phi\left(t_{\lambda}\right)$. Moreover, a sufficient condition for the existence of $t_{\lambda}=$ $Q^{-1}(\lambda)$ is that $\phi(t)<\infty$ for all real $t$ and $P\left(X_{1}>\lambda\right)>0$. Now, if $P\left(X_{1}>\lambda\right)=$ 0 , particularly if $\lambda>\mu$, the essential supremum of $X_{1}$, then $\Sigma(N, K) \leq \mu<\lambda$ for all $N$ and $K$ so that $\lim _{N \rightarrow \infty} \Sigma(N, K)=\lambda$ is not possible. In our case, on the other hand, $\Sigma_{r}(N, K)>\lambda$ is possible even if $\lambda>\mu$, so no such condition relating $\phi$ and $\lambda$ is necessary.

We consider a sequence of numbers $\left\{z_{n}: 1 \leq n<\infty\right\}$ such that $z_{n} \rightarrow \infty$ and $n^{-1 / 2} z_{n} \rightarrow 0$ as $n \rightarrow \infty$, and we want to study the probability $P\left(n^{-1 / 2} S_{n} \geq z_{n}\right)$, where $S_{n}=\Sigma_{k=1}^{n} X_{k}$. We will be especially interested in the case $z_{n}=$ $\lambda n^{\alpha / 2}$, where $\alpha=(2-r) / r$ for $1<r<2$. In the case $r=2, \alpha=2$ and we know by the most elementary form of the central limit theorem that

$$
P\left(n^{-1 / 2} S_{n} \geq \lambda\right) \rightarrow 1-\Phi(\lambda)=(2 \pi)^{-1 / 2} \int_{\lambda}^{\infty} \exp \left(-u^{2} / 2\right) d u \quad \text { as } n \rightarrow \infty .
$$

For $r<2, \alpha>0$ so that $P\left(n^{-1 / 2} S_{n} \geq \lambda n^{a / 2}\right)$ tends to 0 as $n \rightarrow \infty$. The rate at which this probability tends to 0 when $r=1$ is the subject of Cramér [3] and Bahadur and Ranga Rao [1]. We now deal with the case $1<r<2$.

Under the conditions discussed above, we have the following large deviation theorem, which can be found in $[6$, p. 171]:

Theorem 1.1 (Petrov). If $\phi(t)<\infty$ for $|t|<B$, where $0<B \leq \infty$, and $z_{n} \rightarrow \infty$ and $n^{-1 / 2} z_{n} \rightarrow 0$ as $n \rightarrow \infty$, then 
where

$$
P\left(n^{-1 / 2} S_{n} \geq z_{n}\right)=\left\{1-\Phi\left(z_{n}\right)\right\} \exp \left\{n^{-1 / 2} z_{n}^{3} \lambda\left(n^{-1 / 2} z_{n}\right)\right\}\left\{1+O\left(n^{-1 / 2} z_{n}\right)\right\},
$$

$$
\Phi(z)=(2 \pi)^{-1 / 2} \int_{-\infty}^{z} \exp \left(-t^{2} / 2\right) d t
$$

and $\lambda(z)$ is the Cramér series.

In Petrov's theorem, the Cramér series $\lambda(z)=\Sigma_{k=0}^{\infty} \lambda_{k} z^{k}$ is a power series whose coefficients depend on the moments and semi-invariants of $X_{1}$ and which converges for all sufficiently small values of $z$, the radius of cono vergence depending on $\phi$. Further details about the series are available, for example, in Cramér's original article [3] and in Chapter 7 of Ibragimov and Linnik's book [6]. In addition, by a fact proved in Feller's text [5, p. $175], z_{n} \rightarrow \infty$ implies that

$$
1-\Phi\left(z_{n}\right)=(2 \pi)^{-1 / 2} z_{n}^{-1} \exp \left(-z_{n}^{2} / 2\right)\{1+o(1)\}
$$

as $n \rightarrow \infty$. Petrov's the orem then yields

Corollary 1.2. For $1<r<2$ and $\alpha=(2-r) / r$, as $n \rightarrow \infty$,

$$
\begin{aligned}
P\left(S_{n} \geq \beta n^{1 / r}\right)= & \left(2 \pi \beta^{2} n^{\alpha}\right)^{-1 / 2} \\
& \cdot \exp \left\{-1 / 2 \beta^{2} n^{\alpha}\left(1-\beta n^{(\alpha-1) / 2} \lambda\left(\beta n^{(a-1) / 2}\right)\right)\right\}(1+o(1)) .
\end{aligned}
$$

Proof. In Theorem 1.1 and (*) above, we take $z_{n}=\beta n^{(2-r) / 2 r}=\beta n^{\alpha / 2}$, where $0<\alpha<1$. Therefore $z_{n}^{-1}=\beta^{-1} n^{-\alpha / 2}, z_{n}^{2}=\beta^{2} n^{a}, z_{n}^{3}=\beta^{3} n^{3 a / 2}$, and $n^{-1 / 2} z_{n}=\beta n^{(a-1) / 2} \quad 0$ as $n \rightarrow \infty$. We can therefore write that

$$
P\left(S_{n} \geq \beta n^{1 / r}\right)=P\left(n^{-1 / 2} S_{n} \geq \beta n^{a / 2}\right) \text {. }
$$

The result follows by inserting (*) into the conclusion of Theorem 1.1 and then making the substitutions for $z_{n}$.

The following corollary expresses the large deviation result in the form needed for the proof of the main theorem:

Corollary 1.3. If $\phi(t)<\infty$ for $|t|<B$, where $0<B \leq \infty$, then for all $\beta>0$ and all sufficiently large $n$, there exist numbers $\theta_{n}$ depending on $\lambda$ and $\phi$ such that $\theta_{n} \rightarrow 0$ as $n \rightarrow \infty$ and

$$
\begin{aligned}
\exp \left\{-1 / 2 \beta^{2} n^{\alpha}\left(1+\left|\theta_{n}\right|\right)\right\} & \leq 2\left(2 \pi \beta^{2} n^{\alpha}\right)^{1 / 2} P\left(n^{-1 / r} S_{n} \geq \beta\right) \\
& \leq 3 \exp \left\{-1 / 2 \beta^{2} n^{\alpha}\left(1-\left|\theta_{n}\right|\right)\right\}
\end{aligned}
$$

where $1<r<2$ and $\alpha=(2-r) / r$. 
Proof. We apply Corollary 1.2, noting that $0<\alpha<1$ and that $\lambda(z)$ converges for all sufficiently small $z$. We then set

$$
\theta_{n}=\beta n^{(a-1) / 2} \lambda\left(\beta n^{(a-1) / 2}\right),
$$

and we take $n$ large enough so that $0.5<1+o(1)<1.5$.

2. The law of large numbers. This section contains the proof of the main theorem of this article, the extension of the Erdös-Rényi new law of large numbers. For $1<r<2$ and $S_{n}=\Sigma_{k=1}^{n} X_{k}$, we define

$$
\Sigma_{r}(N, K)=\max \left\{K^{-1 / r}\left(S_{n+K}-S_{n}\right): 0 \leq n \leq N-K\right\} \text {. }
$$

We now state and prove the main theorem:

Theorem 2.1. If $\left\{X_{n}: 1 \leq n<\infty\right\}$ is a sequence of $i . i . d$. random variables with $E\left(X_{1}\right)=0, \operatorname{Var}\left(X_{1}\right)=1$, and $m_{\circ} g \circ \circ \phi(t)<\infty$ for $|t|<B$, where $0<B \leq \infty$, and $1<r<2$, then, for every $\lambda>0$,

$$
P\left\{\lim _{N \rightarrow \infty} \Sigma_{r}\left(N,\left[\left(2 \lambda^{-2} \log N\right)^{1 / a}\right]\right)=\lambda\right\}=1,
$$

where $\alpha=(2-r) / r$.

Proof. We take $K_{N}=\left[\left(2 \lambda^{-2} \log N\right)^{1 / a}\right]$, and to simplify the notation, we set

$$
\Sigma(r, \lambda, N)=\Sigma_{r}\left(N, K_{N}\right) \text { and } S_{N}(n, \lambda)=K_{N}^{-1 / r}\left(S_{n+K_{N}}-S_{n}\right) .
$$

For $\epsilon>0$ arbitrary, we define $\lambda^{\prime \prime}=\lambda+\epsilon$, and we obtain from Corollary 1.3 that

$$
\begin{aligned}
P(\Sigma(r, \lambda, N) & \left.\geq \lambda^{\prime \prime}\right)=P\left(\max _{0 \leq n \leq N-K_{N}} S_{N}(n, \lambda) \geq \lambda^{\prime \prime}\right) \\
& \leq \sum_{n=0}^{N-K_{N}} P\left(S_{N}(n, \lambda) \geq \lambda^{\prime \prime}\right) \\
& \leq\left(N-K_{N}+1\right) 3\left(2 \lambda^{\prime \prime}\right)^{-1}\left(2 \pi K_{N}^{a}\right)^{-1 / 2} \exp \left\{-1 / 2\left(\lambda^{\prime \prime}\right)^{2} K_{N}^{a}\left(1-\left|\theta_{K_{N}}^{\prime \prime}\right|\right)\right\},
\end{aligned}
$$

where $\theta_{n}^{\prime \prime}=\theta_{n}\left(\lambda^{\prime \prime}\right)$. We can choose $N^{\prime \prime}$ so large that, for $N \geq N^{\prime \prime}$,

$$
\begin{aligned}
1 / 2\left(\lambda^{\prime \prime}\right)^{2} K_{N}^{a}\left(1-\left|\theta_{K_{N}}^{\prime \prime}\right|\right) & =1 / 2 \lambda^{2}\left(1+\lambda^{-1} \epsilon\right)^{2}\left\{K_{N}\left(K_{N}+1\right)^{-1}\right\}^{\alpha}\left(K_{N}+1\right)^{\alpha}\left(1-\left|\theta_{K_{N}}^{\prime \prime}\right|\right) \\
& \geq 1 / 2 \lambda^{2}\left(1+\delta_{1}\right) 2 \lambda^{-2} \log N=\left(1+\delta_{1}\right) \log N
\end{aligned}
$$


as $K_{N}+1>\left(2 \lambda^{-2} \log N\right)^{1 / \alpha}$, where $\delta_{1}>0$ is a constant depending only on $\lambda$ and $\epsilon$. Increasing $N^{\prime \prime}$, if necessary, so that also $\left\{K_{N}\left(K_{N}+1\right)^{-1}\right\}^{a}>1 / 2$ for $N \geq N^{\prime \prime}$, we have that

$$
\begin{aligned}
P\left(\Sigma(r, \lambda, N) \geq \lambda^{\prime \prime}\right) & \leq 3 N(2 \lambda)^{-1}\left(4 \pi \lambda^{-2} \log N\right)^{-1 / 2} \exp \left\{-\left(1+\delta_{1}\right) \log N\right\} \\
& \leq 3(\log N)^{-1 / 2} N^{-\delta} 1 \leq 3 N^{-\delta} 1 .
\end{aligned}
$$

For all large values of $N$ such that $\left[\left(2 \lambda^{-2} \log N\right)^{1 / a}\right]=j$, we have $j \leq$ $\left(2 \lambda^{-2} \log N\right)^{1 / a}<j+1$, so that

$$
\exp \left\{1 / 2 \lambda^{2} j^{\alpha}\right\} \leq N<\exp \left\{1 / 2 \lambda^{2}(j+1)^{\alpha}\right\} .
$$

We denote $\left[\exp \left(1 / 2 \lambda^{2}{ }_{j}^{a}\right)\right]$ by $E_{j}$, and then we define $N_{j}$ to be the largest integer such that $\left[\left(2 \lambda^{-2} \log N\right)^{1 / a}\right]=j$, so that $N_{j} \geq E_{j}$. It follows that, for $j^{\prime \prime}=\left[\left(2 \lambda^{-2} \log N^{\prime \prime}\right)^{1 / a}\right]$,

$$
\sum_{j=j^{\prime \prime}}^{\infty} P\left(\Sigma_{r}\left(N_{j}, j\right) \geq \lambda^{\prime \prime}\right) \leq \sum_{j=j^{\prime \prime}}^{\infty} 3 N_{j}^{-\delta} 1 \leq 3 \sum_{j=j^{\prime \prime}}^{\infty} E_{j}^{-\delta} 1<\infty,
$$

since the series converges by the integral test. Therefore, by the BorelCantelli lemma, the probability is one that $\Sigma_{r}\left(N_{j}, j\right)<\lambda^{\prime \prime}$ for all but finitely many values of $j$. But, for each $N$, such that $N_{j-1}<N \leq N_{j}$, we have $\Sigma_{r}(N, j) \leq \Sigma_{r}\left(N_{j}, j\right)$ because $N \leq N_{j}$, so that $\Sigma_{r}(N, j) \geq \lambda^{\prime \prime}$ implies $\Sigma_{r}\left(N_{j}, j\right) \geq$ $\lambda^{\prime \prime}$ as well. Therefore the probability is one that $\Sigma_{r}(N, j)<\lambda^{\prime \prime}$ for all but finitely many values of $j$, where $j=K_{N}=\left[\left(2 \lambda^{-2} \log N\right)^{1 / a}\right]$ 。 It follows that

$$
P\left\{\limsup _{N \rightarrow \infty} \Sigma_{r}\left(N,\left[\left(2 \lambda^{-2} \log N\right)^{1 / a}\right]\right)<\lambda+\epsilon\right\}=1,
$$

and, since $\epsilon>0$ is arbitrary, we have

$$
P\left\{\limsup _{N \rightarrow \infty} \Sigma_{r}\left(N,\left[\left(2 \lambda^{-2} \log N\right)^{1 / / \alpha}\right]\right) \leq \lambda\right\}=1 .
$$

On the other hand, we take $\epsilon>0$ arbitrary and define $\lambda^{\prime}=\lambda-\epsilon$. Then, using $S_{N}[m, \lambda]$ to denote $K_{N}^{-1 / r}\left(S_{(m+1) K_{N}}-S_{m K_{N}}\right)$ for integer values of $m$, we have that

$$
\begin{aligned}
P\left\{\Sigma(r, \lambda, N)<\lambda^{\prime}\right\} & =P\left\{\max _{0 \leq n \leq N-K_{N}} S_{N}(n, \lambda)<\lambda^{\prime}\right\} \\
& \leq P\left\{S_{N}[m, \lambda]<\lambda^{\prime} \text { for } 0 \leq m \leq\left[N K_{N}^{-1}\right]-1\right\}
\end{aligned}
$$

since $m K_{N}=n$ and $0 \leq n \leq N-K_{N}$ imply that $0 \leq m K_{N} \leq N-K_{N}$, and $(m+1) K_{N}=N$ if and only if $m=N K_{N}^{-1}-1$. By independence of the incre- 
ments, we have

$$
P\left\{\Sigma(r, \lambda, N)<\lambda^{\prime}\right\} \leq \prod_{m=0}^{\left[N K_{N}^{-1}\right]-1} P\left\{S_{N}[m, \lambda]<\lambda^{\prime}\right\} \leq\left\{P\left(K_{N}^{-1 / r} S_{K_{N}}<\lambda^{\prime}\right)\right\}^{\left[N K_{N}^{-1}\right]} .
$$

From Corollary 1.3, we can write that

$$
\begin{aligned}
P\left\{K_{N}^{-1 / r} S_{K_{N}}<\lambda^{\prime}\right\} & =1-P\left\{S_{K_{N}} \geq \lambda^{\prime} K_{N}^{1 / r}\right\} \\
& \leq 1-\left(2 \lambda^{\prime}\right)^{-1}\left(2 \pi K_{N}^{a}\right)^{-1 / 2} \exp \left\{-1 / 2\left(\lambda^{\prime}\right)^{2} K_{N}^{a}\left(1+\left|\theta_{K_{N}}^{\prime}\right|\right)\right\},
\end{aligned}
$$

where $\theta_{n}^{\prime}=\theta_{n}\left(\lambda^{\prime}\right)$. Now $\lambda^{\prime}=\lambda-\epsilon$ and $K_{N} \leq\left(2 \lambda^{-2} \log N\right)^{1 / a}$ imply that

$$
\begin{aligned}
1 / 2\left(\lambda^{\prime}\right)^{2} K_{N}^{\alpha}\left(1+\left|\theta_{K_{N}}^{\prime}\right|\right) & \leq 1 / 2 \lambda^{2}\left(1-\epsilon \lambda^{-1}\right)^{2}\left(2 \lambda^{-2} \log N\right)\left(1+\left|\theta_{K_{N}}^{\prime}\right|\right) \\
& \leq\left(1-\epsilon \lambda^{-1}\right)(\log N)\left(1+\left|\theta_{K_{N}}^{\prime}\right|\right) .
\end{aligned}
$$

We can choose $N^{\prime}$ so large that $\left(1-\epsilon \lambda^{-1}\right)\left(1+\left|\theta_{K_{N}}^{\prime}\right|\right)<1-3 \delta_{2}$, where $\delta_{2}>0$ is a constant depending only on $\lambda$ and $\epsilon$. Therefore

$$
\begin{aligned}
P\left\{K_{N}^{-1 / r} S_{K_{N}}<\lambda^{\prime}\right\} & \leq 1-(2 \lambda)^{-1}\left(2 \pi 2 \lambda^{-2} \log N\right)^{-1 / 2} N^{-\left(1-3 \delta_{2}\right)} \\
& \leq 1-(16 \pi \log N)^{-1 / 2} N^{-\left(1-3 \delta_{2}\right)} \\
& \leq 1-N^{-\left(1-2 \delta_{2}\right)} \leq \exp \left\{-N^{-\left(1-2 \delta_{2}\right)}\right\},
\end{aligned}
$$

taking $N^{\prime}$ also large enough so that $(16 \pi \log N)^{-1 / 2} \geq N^{-\delta}$. It follows that, for all $N \geq N^{\prime}$,

$$
P\left\{\Sigma(r, \lambda, N)<\lambda^{\prime}\right\} \leq \exp \left\{-\left[N K_{N}^{-1}\right]_{N}-(1-2 \delta 2)\right\} \leq \exp \left\{-N^{\delta}{ }^{2}\right\},
$$

if we take $N^{\prime}$ large enough so that $K_{N} \leq N^{\delta}$ and $\left[N K_{N}^{-1}\right] \geq N^{1-\delta_{2}}$. Then

$$
\sum_{N=N^{\prime}}^{\infty} P\left\{\Sigma(r, \lambda, N)<\lambda^{\prime}\right\} \leq \sum_{N=N^{\prime}}^{\infty} \exp \left\{-N^{\delta}\right\}<\infty
$$

by the integral test. Therefore, the Borel-Cantelli lemma asserts that, $w$ ith probability one, only finitely many $\Sigma(r, \lambda, N)$ are less than $\lambda^{\prime}$. Therefore

$$
P\left\{\liminf _{N \rightarrow \infty} \Sigma(r, \lambda, N) \geq \lambda^{\prime}\right\}=1 \text {. }
$$

Since $\lambda^{\prime}=\lambda-\epsilon$, where $\epsilon>0$ is arbitrary, this means that

$$
P\left\{\liminf _{N \rightarrow \infty} \Sigma_{r}\left(N,\left[\left(2 \lambda^{-2} \log N\right)^{1 / a}\right]\right) \geq \lambda\right\}=1,
$$

and the theorem follows. 
3. Comparison with the Erdös-Rényi theorem. The Erdös-Rényi theorem makes the following assertion:

Theorem 3.1 (Erdös-Rényi). If $\left\{X_{n}: 1 \leq n<\infty\right\}$ is a sequence of i.i.d. random variables with $E\left(X_{1}\right)=0, \operatorname{Var}\left(X_{1}\right)=1$, and m.g.f. $\phi(t)<\infty$ for a $|t|<B$, where $0<B \leq \infty$, then, for every $\lambda$ in the range of $Q(t)=\phi^{\prime}(t) / \phi(t)$,

$$
P\left\{\lim _{N \rightarrow \infty} \Sigma_{1}(N,[C(\lambda) \log N])=\lambda\right\}=1,
$$

where $C(\lambda)=\left\{\lambda Q^{-1}(\lambda)-\log \phi\left(Q^{-1}(\lambda)\right)\right\}^{-1}$.

We note that in going from Theorem 2.1 (which holds only for $1<r<2$ ) to The orem 3.1 (which holds only for $r=1$ ), the quantity $2 \lambda^{-2}$ suddenly jumps to $C(\lambda)$. Why does that instantaneous jump occur? We observe that, by L'Hôpital's Rule,

$$
\begin{aligned}
\lim _{\lambda \rightarrow 0} 2 \lambda^{-2} / C(\lambda) & =2 \lim _{\lambda \rightarrow 0} \lambda^{-2}\left\{\lambda Q^{-1}(\lambda)-\log \phi\left(Q^{-1}(\lambda)\right)\right\} \\
& =\lim _{\lambda \rightarrow 0} \lambda^{-1} Q^{-1}(\lambda)=\left(Q^{-1}\right)^{\prime}(0)=1,
\end{aligned}
$$

as $\operatorname{Var}\left(X_{1}\right)=1$. This seems to say that as $\lambda n$ gets closer to $\lambda n^{1 / r}$ (by the process of $\lambda$ tending to 0$), C(\lambda)$ gets closer to $2 \lambda^{-2}$, which would be its actual value if $\lambda n$ really were $\lambda n^{1 / r}$ for $1<r<2$.

In the special case of normally distributed $X_{1}$, it turns out that $C(\lambda)=$ $2 \lambda^{-2}$ for all values of $\lambda$, because $Q(t)=t$ in that case. We have therefore that

$$
P\left\{\lim _{N \rightarrow \infty} \Sigma_{r}\left(N,\left[\left(2 \lambda^{-2} \log N\right)^{1 / \alpha}\right]\right)=\lambda\right\}=1
$$

for $1 \leq r<2$ in the normal case, where $\alpha=(2-r) / r$. In the general case, however, for $r=1$, denoting the value of $C(\lambda)$ in Theorem 3.1 by $2 \theta^{-2}$ so that $\lambda$ is $C^{-1}\left(2 \theta^{-2}\right)$, we can write

$$
P\left\{\lim _{N \rightarrow \infty} \Sigma_{1}\left(N,\left[2 \theta^{-2} \log N\right]\right)=C^{-1}\left(2 \theta^{-2}\right)\right\}=1,
$$

where $x=C^{-1}\left(2 \theta^{-2}\right)$ is the (unique) solution of the equation

$$
x Q^{-1}(x)-\log \phi\left(Q^{-1}(x)\right)=\theta^{2} / 2 \text {. }
$$

The solution exists if $C^{-1}\left(2 \theta^{-2}\right)$ lies in the range of $Q$. Now, because $C(\lambda)$ is a strictly decreasing function of $\lambda$, and $C(\lambda)<2 \lambda^{-2}$ for $\lambda>0$, it follows that $C^{-1}\left(2 \lambda^{-2}\right)>C^{-1}(C(\lambda))=\lambda$. Under the conditions of Theorem 2.1 , we then have $w$ ith probability one that 
and

$$
\lim _{N \rightarrow \infty} \sum_{r}\left(N,\left[\left(2 \lambda^{-2} \log N\right)^{1 / a}\right]\right)=\lambda \quad \text { for } 1<r<2
$$

$$
\lim _{N \rightarrow \infty} \Sigma_{1}\left(N,\left[2 \lambda^{-2} \log N\right]\right)=C^{-1}\left(2 \lambda^{-2}\right) \quad \text { for } r=1
$$

where, in the case of normality, $C^{-1}\left(2 \lambda^{-2}\right)=\lambda$.

4. Acknowledgment. The author would like to express his thanks to a referee who suggested the use of the already existing theorem of Petrov in place of a less general large deviation theorem created expressly for the purpose of proving Corollary 1.3 .

\section{REFERENCES}

1. R. R. Bahadur and R. Ranga Rao, On deviations of the sample mean, Ann. Math. Statist. 31 (1960), 1015-1027. MR 22 \#8549.

2. S. A. Book, The Erdös-Rényi new law of large numbers for weighted sums, Proc. Amer. Math. Soc. 38 (1973), 165-171. MR 46 \#10044.

3. H. Cramér, Sur un nouveau théorème-limite de la théorie des probabilités, Actualités Sci. Indust., no. 736, Hermann, Paris, 1938, pp. 5-23.

4. P. Erdös and A. Rényi, On a new law of large numbers, J. Analy se Math. 23 (1970), 103-111. MR 42 \#6907.

5. W. Feller, An introduction to probability theory and its applications. I, 3rd ed., Wiley, New York, 1968. MR 37 \#3604.

6. I. A. Ibragimov and Yu. V. Linnik, Independent and stationary sequences of random variables, Wolters-Noordhoff, Groningen, 1971.

7. M. Loève, Probability theory, 3rd ed., Van Nostrand, Princeton, N. J., 1963. MR 34 \#3596.

8. V. V. Petrov, Generalization of Cramér's limit theorem, Uspehi Mat. Nauk 9 (1954), no. 4 (62), 195-202. (Russian) MR 16, 378.

DE PARTMENT OF MATHEMATICS, CALIFORNIA STATE COLLEGE, DOMINGUEZ HILLS, CALIF ORNIA 90747 